\title{
Efficacy of acupuncture in improving the symptoms and the quality of life of patients with moderate or severe acne vulgaris: study protocol for a randomized controlled trial
}

Ruimin Jiao ${ }^{1,2+}$, Man Huang ${ }^{1+}$, Weina Zhang ${ }^{1}$ and Zhishun Liu ${ }^{1 *}$

\begin{abstract}
Background: Acne vulgaris (AV) is a common chronic dermatologic disease that tends to impair the appearance and quality of life (QoL) of patients. Although several trials have indicated the effectiveness of acupuncture for treatment of patients with $\mathrm{AV}$, the results of these trials have been contested, owing to potential bias in their design. Thus, there is a lack of robust data to evaluate the efficacy of acupuncture in patients with AV. In addition, none of the previous clinical trials of acupuncture therapy for AV used QoL as a primary outcome or employed a sham acupuncture control arm. The aim of the present study is to evaluate the effectiveness of acupuncture in treating the symptoms and $\mathrm{QoL}$ of patients with moderate or severe AV.

Methods/design: One hundred eligible participants with AV will be randomly assigned to an acupuncture or a sham acupuncture group (1:1 allocation). All participants will receive 4-week treatment comprising a total of 12 sessions (3 sessions per week). The primary outcome will be change from baseline in the Skindex-16 scale total score at treatment completion. The secondary outcomes will be Skindex-16 subscale score, Dermatology Life Quality Index scale total score, total lesion count and inflammatory lesion count, visual analogue scale scores for assessment of itch and pain, patient expectations of acupuncture, and blinding of the effect of sham acupuncture. Follow-up evaluation will be performed at weeks 16 and 28. All outcome analyses will be performed in the intention-to-treat population.
\end{abstract}

Discussion: We expect to evaluate the effectiveness of acupuncture in ameliorating the symptoms and improving the QoL of patients with moderate or severe AV compared with sham acupuncture with more robust evidence. The limitations of the trial design are its single-center scope, relatively small sample size, and lack of blinding of the acupuncturists.

Trial registration: Chinese Clinical Trial Registry, ChiCTR-1900023649. Registered on January 2, 2019.

Keywords: Acupuncture, Acne vulgaris, Quality of life, Randomized controlled trial, Protocol

\footnotetext{
* Correspondence: liuzhishun@aliyun.com

${ }^{\dagger}$ Ruimin Jiao and Man Huang contributed equally to this work.

'Department of Acupuncture, Guang'anmen Hospital, China Academy of

Chinese Medical Sciences, No. 5 Beixiange Street, Xicheng District, Beijing,

China

Full list of author information is available at the end of the article
}

(c) The Author(s). 2020 Open Access This article is licensed under a Creative Commons Attribution 4.0 International License, which permits use, sharing, adaptation, distribution and reproduction in any medium or format, as long as you give appropriate credit to the original author(s) and the source, provide a link to the Creative Commons licence, and indicate if changes were made. The images or other third party material in this article are included in the article's Creative Commons licence, unless indicated otherwise in a credit line to the material. If material is not included in the article's Creative Commons licence and your intended use is not permitted by statutory regulation or exceeds the permitted use, you will need to obtain permission directly from the copyright holder. To view a copy of this licence, visit http://creativecommons.org/licenses/by/4.0/. The Creative Commons Public Domain Dedication waiver (http://creativecommons.org/publicdomain/zero/1.0/) applies to the data made available in this article, unless otherwise stated in a credit line to the data. 


\section{Background}

Acne vulgaris (AV) is a common chronic dermatologic disease involving pilosebaceous units $[1,2]$. Skin lesions are the main symptoms of AV; they typically appear over the face, chest, and back [1,3]. An estimated 79-95\% of the adolescent population is affected by AV [4]. The skin lesions include comedones, erythematous papules, pustules, nodules, deep pustules, and scarring. The occurrence of AV lesions on the face may induce anxiety and decrease self-esteem $[5,6]$, leading to a decline in the quality of life (QoL) [5-8].

The American Society of Dermatology recommends benzoyl peroxide, topical retinoids, or systemic antibiotic therapy as the first-line treatment for mild to severe AV [1-3]. However, these pharmaceutical therapies may cause side effects of drying, peeling, erythema, and skin irritation; in addition, prolonged treatment may result in drug resistance, and the associated recurrence rate is relatively high [9]. Therefore, there is an increasing interest in exploring natural and safe therapies for AV $[9,10]$. These natural and safer treatment options include complementary and alternative treatment remedies, such as herbal medicine and acupuncture $[9,10]$.

Several trials have indicated that acupuncture may alleviate the skin lesions and improve the QoL of patients with AV [11-15]. However, these trials were affected by potential bias due to small sample sizes, nonplacebo/ sham/waiting list control, or use of self-defined outcome measures; thus, they did not provide robust evidence of the efficacy of acupuncture in patients with AV. Therefore, we conducted a pilot trial (unpublished) from April 2017 to March 2018 in which 42 patients with moderate or severe AV were randomly allocated to an acupuncture group $(n=21)$ or a sham acupuncture group $(n=$ $21)$. After a 4 -week treatment, the number of inflammatory lesions in the acupuncture group $(n=21)$ was $35.62 \pm 15.51$, with a reduction of $6.62 \pm 15.42$ from baseline. The number of inflammatory lesions in the sham acupuncture group $(n=21)$ was $30.05 \pm 21.16$, with a reduction of $15.10 \pm 20.13$ from the baseline. The difference between the two groups was $8.48 \pm 7.74$, and there was no significant between-group difference with the $p=0.137$ due to $p>0.05$ was considered nonsignificant. These findings contradict the results of previous trials [11-14], and authors of a systematic review of AV also reported that there was no difference between acupuncture and the waiting list for reducing the number of inflammatory lesions [16]. Unlike drugs, which act directly on the pathogen, acupuncture restores normal functions through motivating or inducing the inherent regulatory system [17]. Therefore, acupuncture may relieve other symptoms of AV (itch or pain) $[18,19]$ and improve the QoL [11]. The Skindex-16 scale is a sensitive and specific tool for assessing the symptoms and
QoL of patients with AV, and it includes seven questions related to the symptoms (itch or pain) and characteristics (skin lesions) of AV [20, 21]. Following the 4-week treatment, the total score on the Skindex-16 scale for acupuncture $(n=21)$ was $19.89 \pm 15.40$, with a reduction of $12.50 \pm 19.09$ from the baseline. The total score on the Skindex-16 scale for sham acupuncture $(n=21)$ was $30.43 \pm 19.39$, with a reduction of $0.40 \pm 21.12$ from the baseline. The difference between the two groups was $12.9 \pm 11$, and there was a significant between-group difference in this instance with the $p=0.044$ due to $p<$ 0.05 was considered statistically significant. Therefore, the results of our pilot study indicated that acupuncture may help to relieve total symptoms and improve the QoL of patients with moderate or severe AV. So far, there are two systematic reviews that recommend using the measure of QoL for evaluating the effect of acupuncture on AV $[10,16]$, but no clinical trials have been conducted that use the measure of QoL to evaluate the effect of acupuncture on the symptoms and the QoL of patients with AV. Therefore, we intend to use the Skindex-16 scale as a primary outcome measure in our planned study to evaluate the effect of acupuncture compared with sham acupuncture on the symptoms and QoL of patients with moderate or severe AV.

\section{Methods/design}

\section{Study design}

The proposed study is a prospective, randomized, sham acupuncture controlled trial with two parallel arms using a 1:1 allocation ratio. The trial will be conducted at the Guang'anmen Hospital, China Academy of Chinese Medical Sciences. The study protocol conforms to the Standard Protocol Items: Recommendations for Interventional Trials (SPIRIT) [22] and the Standards for Reporting Interventions in Clinical Trials of Acupuncture (STRICTA) [23].

\section{Study recruitment}

Participants will be recruited between August 2019 and December 2020 at the Guang' anmen Hospital via advertisements on posters, the WeChat app, and hospital websites. A dermatologist will be responsible for the screening, which will include diagnosis of the type of acne of patients and a series of physical examinations (discrimination between inflammatory and noninflammatory lesions of $\mathrm{AV}$ by the pictures of participants' faces obtained using a digital camera) according to the diagnostic criteria for $\operatorname{AV}[2,24]$ and the classification of lesions of AV [25], respectively.

All participants will be asked by a research assistant to read the informed consent form that describes the trial, randomization process for treatments, and the potential benefits and risks of the trial. Participants will be 
informed that both acupuncture and sham acupuncture may be effective for $\mathrm{AV}$, and they will be randomly assigned to the acupuncture group or the sham acupuncture group. Participants will be allowed to withdraw from the trial at any time. Written informed consent will be obtained from all subjects prior to their enrollment.

The demographic and clinical characteristics of the participants at baseline will be recorded by a research assistant: age, sex, race, body mass index (in $\mathrm{kg} / \mathrm{m}^{2}$ ), marital status, education level (primary education or below, secondary education, and tertiary education), time of sports activity ( $0-3$ times/month, $1-2$ times/ week, 3-4 times/week, and $\geq 5$ times/week), daily sleep duration $(<7 \mathrm{~h}, 7-9 \mathrm{~h}$, and $>9 \mathrm{~h})$, smoking history, history of alcohol intake, AV duration in months, and severity of AV as assessed by Global Acne Grading System (GAGS) score (none [0 score], mild [1-18 score], moderate [19-30 score], severe [31-38 score], and markedly severe [>39 score]) [26].

\section{Randomization and allocation concealment}

The randomization scheme has been prepared by the National Clinical Drug Testing Institute of the Guang'anmen Hospital. Participants will be randomly assigned to receive acupuncture or sham acupuncture treatment in a 1:1 ratio with the fixed block of 4 . Sealed opaque envelopes will be used to ensure randomization concealment. The number of the randomization sequence and information on group allocation will be sealed in ordered envelopes. With the inclusion of patients, those envelopes will be opened one by one in sequence. The envelopes will be kept by a research assistant who is not involved in the treatment or assessment. Additionally, assistant researchers will perform a baseline assessment of all participants ( 1 week to 0 week) prior to randomization.

\section{Blinding}

The participants, outcome assessors, and statisticians will be blinded to the group allocation. However, because of the characteristics of acupuncture, acupuncturists will not be blinded in this trial. To assess the blinding effect of sham acupuncture, participants will be asked to answer the following questions within $5 \mathrm{~min}$ after any treatment in week 4: "Do you think you have received traditional acupuncture?" The response options will be "yes," "no," or "unclear." The study protocol is illustrated in Fig. 1.

\section{Ethical approval}

This clinical trial will adhere to the principles of the Declaration of Helsinki and has been approved by the Ethics Committee of the Guang'anmen Hospital (2018137-KY-01) (see Additional file 1).

\section{Participants}

One hundred eligible participants with AV will be recruited in the trial.

\section{Inclusion criteria}

1. Individuals aged between 18 and 48 years who qualify for the diagnostic criteria for AV [2, 24]

2. GAGS score between 19 and 38 [26]

\section{Exclusion criteria}

Participants with any of the following conditions will be excluded:

1. Individuals with polymerization acne, explosive acne, drug acne, premenstrual acne, cosmetic acne, occupational acne, or any other subtypes of acne

2. Individuals with other severe diseases that may affect acne, such as polycystic ovary syndrome, thyroid disease, or atypical congenital adrenal hyperplasia

3. Individuals with other skin diseases that may influence the assessment of $\mathrm{AV}$, such as rosacea, folliculitis, or other skin diseases

4. Individuals who have received antibiotics, retinoic acid, steroids, or anti-inflammatory drugs in the preceding 1 month

5. Individuals with severe heart, liver, kidney, hematopoietic system or autoimmune disorders, or severe systemic malnutrition

6. Pregnant and lactating women or those planning to conceive within 12 months

7. Individuals who have received acupuncture treatment in the past 3 months

\section{Intervention}

All participants will receive treatment for 4 weeks with three sessions per week (ideally every other day), for a total of 12 sessions. Acupuncturists with an undergraduate degree or above and clinical experience of over 1 year at the Guang'anmen Hospital will be responsible for treatment. Participants will be treated separately to avoid communication during the trial period. We will discourage all included participants from receiving any other treatment for AV during the study period. If participants receive any other treatment for $\mathrm{AV}$, they will also be included in this study, and all details of the other treatment for AV will be recorded in the case report form. We will compare the proportion of participants using other treatments between the two groups.

\section{Acupuncture group}

The location of acupoints is described as per the Nomenclature and Location of Acupuncture Points 


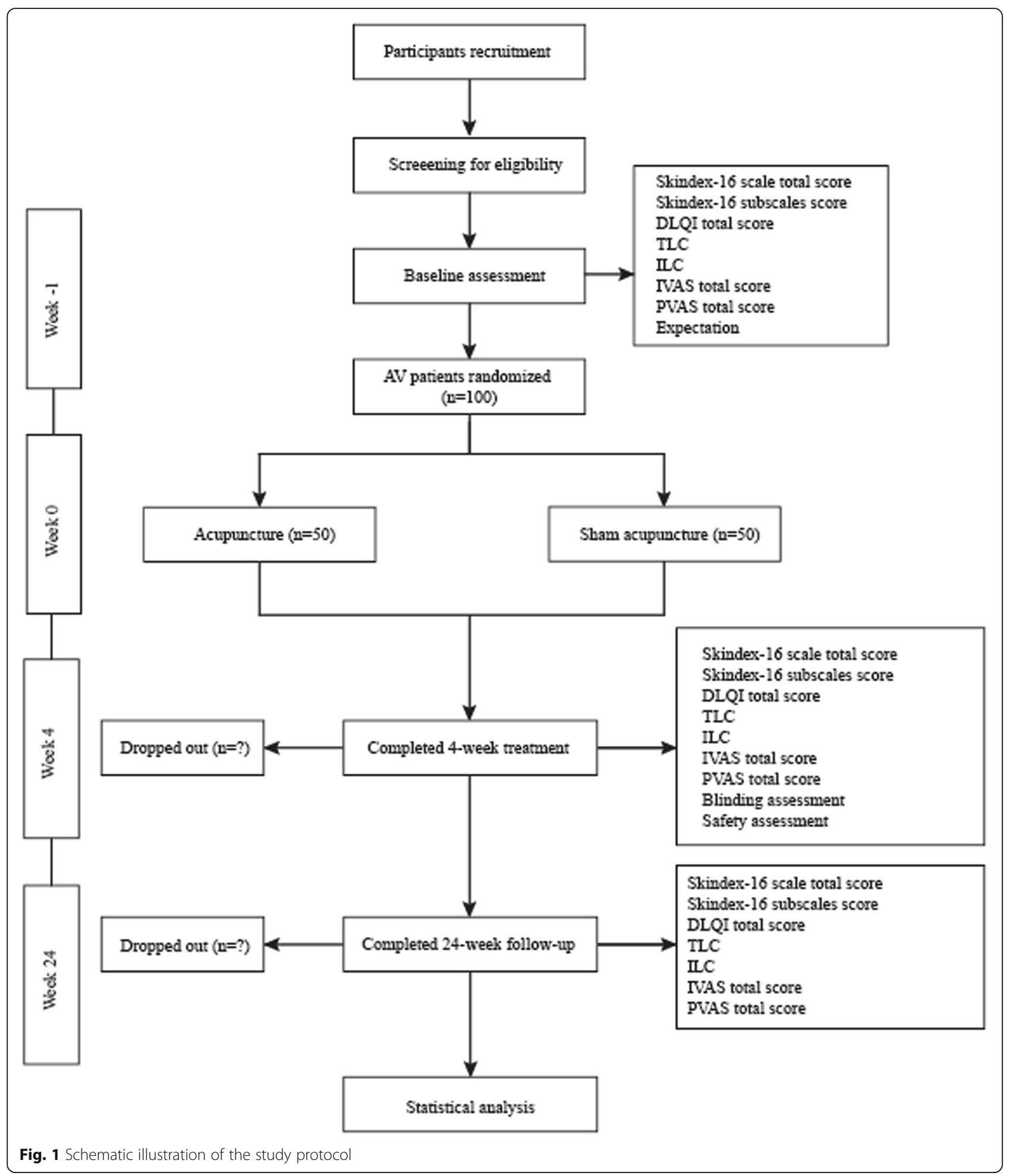

(National Standard of People's Republic of China, 2006 [GB/T 12346-2006]) [27]. The selection of acupoints will be decided with reference to the Guidelines for AV treatment in China (revised version 2014) [28]. Participants in the acupuncture group will receive stimulation at Dazhui (CV14), bilateral Quchi (LI11), bilateral Hegu
(LI4), bilateral Zusanli (ST36), and bilateral Neiting (ST44) with disposable acupuncture needles $(0.30 \times 40$ mm, Huatuo Brand; Suzhou Medical Appliance, Suzhou, Jiangsu, China). After routine disinfection, acupuncture needles will be inserted obliquely into CV14 to a depth of 30-40 mm at an angle of 15-30 degrees; acupuncture 
needles will be vertically inserted into bilateral LI11, bilateral LI4, bilateral ST36, and bilateral ST44 to a depth of 25-30 mm three times (once every $10 \mathrm{~min}$ ) with slight lifting, thrusting, and twisting manipulations to produce a sensation of de-qi. Each session of acupuncture will last $30 \mathrm{~min}$.

\section{Sham acupuncture group}

Participants in sham acupuncture group will receive sham acupuncture at sham CV14 (10 mm to CV14), LI11 (10 mm to LI11), LI4 (10 mm to LI4), ST36 (25 mm to ST36), and ST44 (10 $\mathrm{mm}$ to ST44) with disposable acupuncture needles $(0.30 \times 25 \mathrm{~mm}$, Huatuo Brand $)$. Sham CV14, LI11, LI4, ST36, and ST44 will be vertically inserted to a depth of $1-2 \mathrm{~mm}$ without any manipulation and de-qi. The treatment sessions will last $30 \mathrm{~min}$.

Participants will not be allowed use of other treatments for AV throughout the trial. Detailed information pertaining to use of other treatments will be recorded in the case report form.

\section{Rescue medication}

Participants will not be encouraged to receive any other treatment or medication during the study period to prevent any influence on the results. However, in case of deterioration of the condition of AV during the 4-week treatment period and the 24-week follow-up, oral minocycline hydrochloride capsules will be prescribed to the patients (100 mg per day for 7 days) to relieve the symptoms of pain or itching. Details of the medication used will be recorded in the case report form. The proportion of participants using rescue drugs in the groups will be compared.

\section{Outcome measures}

The primary outcome will be the change in the total score of the Skindex-16 scale from baseline at the end of 4-week treatment. The Skindex-16 scale is a brief, skin-related QoL scale with satisfactory reliability and validity $[20,21]$. It is used to evaluate the efficacy of acupuncture in improving the QoL of patients with AV $[20,21]$. The scale includes a total of 16 items that are categorized into three domains: the symptoms of participants with $\mathrm{AV}$, the emotions of participants with $\mathrm{AV}$, and function of participants with AV $[20,21]$. The Skindex-16 scale score ranges from 0 (best) to 100 (worst), with a minimal clinically important difference (MCID) as $10[20,21]$.

\section{Secondary outcomes}

1. The change from baseline in the Skindex-16 scale total score at weeks 16 and 28
2. The change from baseline in the Skindex-16 subscale (the symptoms of participants with $\mathrm{AV}$, the emotions of participants with AV, and functioning of participants with AV) scores at weeks 4, 16 , and 28

3. The change from baseline in the Dermatology Life Quality Index (DLQI) [29] scale total score at weeks 16 and 28. The DLQI scale is a tool used to assess the health-related QoL of patients with skin diseases. It has a total of ten items. The total score of DLQI ranges from 0 (best) to 30 (worst), with 4 as the MCID in inflammatory skin diseases [30].

4. The change from baseline in the total lesion (inflammatory and noninflammatory lesions) count (TLC) [31, 32] at weeks 4,16 , and 28

5. The change from baseline in the inflammatory lesion count (ILC) [26] at weeks 4, 16, and 28. Inflammatory lesions include inflammatory papules, pustules, and cysts. Noninflammatory lesions include blackhead and whitehead comedones. The lesions are assessed on the forehead, cheeks, nose, and chin. The inflammatory and noninflammatory lesions are counted from the pictures of the face obtained by a dermatologist using a digital camera.

6. The change from baseline in the degree of itch assessed using the Itch Assessment with Visual Analogue Scale (IVAS) [32] at weeks 4, 16, and 28

7. The change from baseline in the severity of pain assessed by visual analogue scale (PVAS) [33] at weeks 4,16 , and 28

8. The participants' expectations of acupuncture will be assessed at baseline using the following two questions: "Do you think acupuncture will be effective for treating the illness?" and "Do you think acupuncture will be effective for relieving the related symptoms of AV?" The response options will be "yes," "no," or "unclear."

The details of the evaluation of outcomes are shown in Table 1.

\section{Safety evaluation}

Adverse events (AEs), including AEs related to acupuncture (broken needle, local hematoma, infection, abscess, and others), AEs related to postacupuncture (nausea, vomiting, palpitations, dizziness, headache, insomnia, or any other symptoms after acupuncture treatment), and AEs unrelated to treatment will be recorded. For safety assessment, details of all AEs will be recorded by a research assistant in the case report form. Any serious adverse events will be reported within $24 \mathrm{~h}$ to the Ethics Approval Committee of the Guang'anmen Hospital of China Academy of Chinese Medical Sciences. The Department of Acupuncture of the Guang'anmen 
Table 1 Time points for assessment of outcomes

\begin{tabular}{|c|c|c|c|c|c|c|c|}
\hline \multirow[t]{2}{*}{ Outcome measures } & \multirow{2}{*}{$\begin{array}{l}\text { Enrollment and allocation } \\
\text { Baseline }\end{array}$} & \multicolumn{4}{|c|}{ Treatment } & \multicolumn{2}{|c|}{ Follow-up } \\
\hline & & Week 1 & Week 2 & Week 3 & Week 4 & Week 16 & Week 28 \\
\hline Skindex-16 total score & $x$ & & & & $x$ & $x$ & $x$ \\
\hline Skindex-16 subscales score & $x$ & & & & $x$ & $x$ & $x$ \\
\hline DLQI total score & $x$ & & & & $x$ & $x$ & $x$ \\
\hline TLC & $x$ & & & & $x$ & $x$ & $x$ \\
\hline ILC & $x$ & & & & $x$ & $x$ & $x$ \\
\hline IVAS score & $x$ & & & & $x$ & $x$ & $x$ \\
\hline PVAS score & $x$ & & & & $x$ & $x$ & $x$ \\
\hline Blinding assessment & & & & & $x$ & & \\
\hline Expectation & $x$ & & & & & & \\
\hline Safety assessment & & $x$ & $x$ & $x$ & $x$ & & \\
\hline
\end{tabular}

Abbreviations: DLQI Dermatology Life Quality Index scale, ILC Inflammatory lesion count, IVAS Itch assessment with visual analogue scale, PVAS Pain assessment with visual analogue scale, TLC Total lesion count

Hospital of China will provide insurance coverage to compensate for any injuries related to the interventions during this study.

\section{Sample size and statistical analysis}

The sample size of this trial was calculated on the basis of primary outcome, which is the change from baseline in the Skindex-16 scale score at the end of week 4. In our unpublished pilot trial, the mean $( \pm$ standard deviation) reductions in the Skindex-16 scale score after 4-week treatment in the acupuncture and sham acupuncture groups were $12.50 \pm 19.09$ and $0.40 \pm 21.12$, respectively. Assuming an alpha risk of $5 \%$ and a beta risk of 20\%, a sample size of 100 (50 participants in each group) was calculated, considering a $20 \%$ dropout rate.

The data will be analyzed using IBM SPSS Statistics version 20.0 software (IBM Corp, Armonk, NY, USA) according to the intention-to-treat principle. Normally distributed continuous variables will be reported as mean \pm standard deviation or $95 \%$ confidence intervals; non-normally distributed continuous variables will be reported as median (interquartile range). Categorical variables will be presented as frequency (percent). For data pertaining to dropouts, the actual observational value of the last observation will be used for statistical analysis. Between-group differences with respect to normally distributed continuous variables will be assessed using analysis of variance, and those with respect to non-normally distributed continuous variables will be assessed using nonparametric tests for the primary outcome and the secondary outcomes. Data pertaining to the participants' expectations of acupuncture and the safety evaluation will be analyzed using the chi-square test or Fisher's exact test. For the blinding assessment, the percentage of participants choosing traditional acupuncture will be assessed using the chi-square test. All $P$ values will be two-tailed; $P<0.05$ will be considered indicative of statistical significance.

\section{Quality control}

All researchers will take a training course before the beginning of this trial. Due measures will be implemented to ensure the traceability and confidentiality of the case report form, informed consent form, and other original data. A double-input method will be used for data entry. AEs will be recorded in detail, properly handled, and tracked. All trial-related procedures and data management will be supervised. The Data Monitoring Committee of the Guang'anmen Hospital will regularly monitor the recruitment and screening of participants, data collection, monitoring, and verification of AEs to ensure that the study is conducted in accordance with the approved protocol.

\section{Discussion}

$\mathrm{AV}$ is a common chronic inflammatory dermatologic disease. It may affect the physical and mental health of patients and may adversely affect their QoL [34]. According to an epidemiological study, approximately $20 \%$ of young people are affected by moderate to severe AV [35]. Several studies have demonstrated a correlation between the QoL and the severity of dermatologic disease [36]. For this reason, this study will only include participants with moderate or severe AV.

The primary outcome of the study will be the Skindex-16 scale score. It is a validated and sensitive scale to measure the QoL of patients with dermatologic diseases [20, 21]. Because lesions of AV mainly occur on areas of the face and are accompanied by other symptoms of itch and pain [32, 33], patients tend to present with psychosocial symptoms and impaired QoL. The Skindex-16 scale consists of 16 questions in the 
symptoms, emotion, and function domains; responses to each question are scored on a 7-point Likert scale [20, 21]. This scale includes seven questions related to the symptoms (itch or pain) and characteristics (lesions) of AV. It is a sensitive and specific tool to assess the symptoms and QoL of patients with moderate or severe AV. Moreover, we also used the DLQI scale to evaluate QoL. It is a brief, skin-related QoL scale with satisfactory reliability and validity, and it was used to further evaluate the effectiveness of acupuncture in improving the QoL of patients with AV [29]. In addition, TLC, ILC, IVAS, and PVAS will also be used as secondary outcomes to assess the lesion counts and main symptoms (itch or pain) related to AV. These would provide a comprehensive evaluation of the overall effectiveness of acupuncture.

In this trial, we aim to evaluate the therapeutic effect of acupuncture on the symptoms and QoL of patients with moderate or severe AV. The possible placebo effects of acupuncture may be attributable to the participants' expectations of acupuncture; the degree of trust between the acupuncturist and the participant; and any difference in the type, frequency, or course of the intervention of acupuncture $[37,38]$. To partially exclude the placebo effects and demonstrate the real effect of acupuncture, we plan to use sham acupuncture as a comparator.

The sham acupuncture is a crucial tool that can minimize participants' expectations and the bias of both participants and researchers and assist in the enforcement of blinding [38]. Use of the non-insertion-type needle on nonacupoints was shown to be an optimal method to reduce the possible biological effects [39]. However, the non-insertion-type needle leaves no marks or wounds on the skin. In this condition, the lack of any needle marks may compromise the blinding of participants. Therefore, we will opt for nonacupoints and minimal acupuncture as the sham acupuncture in this trial. Nonacupoints are in proximity to the classical acupoints, which will increase the feasibility of blinding [38]. Meanwhile, using the nonacupoints and minimal acupuncture without any manipulation may minimize actual needle sensation to mitigate the potential physiologic stimulus $[38,40]$. The outcome assessors of this study will be blinded to the group allocation, which will also mitigate the lack of acupuncturist blinding.

However, some limitations of our study should be acknowledged. First, the single-center scope of the study and the relatively small sample size may lead to overestimation of the effects of acupuncture. Second, because of the characteristics of acupuncture, the acupuncturist will not be blinded in our trial, which may cause potential bias. Last, it is difficult for the sham acupuncture to be as inert as desired in this trial, owing to a slight stimulus or a stronger stimulus of gentle touch possibly leading to different degrees of neurological responses related to the treatment effect $[39,41]$. So, the use of nonacupoints and minimal acupuncture without any manipulation may cause some biological effect leading to falsenegative results [36].

\section{Trial status}

No recruitment currently.

\section{Supplementary information}

Supplementary information accompanies this paper at https://doi.org/10. 1186/s13063-020-04346-7.

Additional file 1. Ethics approval of Guang'anmen Hospital of China Academy of Chinese Medical Sciences.

\section{Abbreviations}

AE: Adverse event; AV: Acne vulgaris; DLQI: Dermatology Life Quality Index: GAGS: Global Acne Grading System; ILC: Inflammatory lesion count; IVAS: Itch Assessment with Visual Analogue Scale; MCID: Minimal clinically important difference; PVAS: Pain Assessment with Visual Analogue Scale; QoL: Quality of life; SPIRIT: Standard Protocol Items: Recommendations for Interventional Trials; STRICTA: Standards for Reporting Interventions in Clinical Trials of Acupuncture; TLC: Total lesion count

\section{Acknowledgements}

Not applicable.

Ethical approval document

Ethics approval of acne vulgaris

\section{Authors' contributions}

$\mathrm{ZL}$ and RJ conceived the idea of this trial and the design of this study. $\mathrm{MH}$ and $\mathrm{WZ}$ are responsible for statistical analysis. RJ, WZ, and $\mathrm{MH}$ are responsible for the recruitment and treatment of participants. RJ drafted the manuscript. ZL revised the manuscript. All authors read and approved the final manuscript.

\section{Funding}

This trial will be conducted without any external funding or internal funding, and all researchers and participants will voluntarily participate in this trial.

\section{Availability of data and materials}

All relevant data will be shared for a period beginning 3 months after publication and ending 5 years after publication.

\section{Ethics approval and consent to participate}

The study protocol has been approved by the Institutional Review Board of the Guang'anmen Hospital in China (approval no. 2018-137-KY-01) (see Additional file 1). All investigators will comply with the principles of the Declaration of Helsinki. All study participants will be voluntarily enrolled after screening, and written informed consent will be obtained prior to enrollment. All study participants will have a discussion with the researchers about the procedure, treatment, and possible risks and benefits of the trial. Moreover, all participants will have the right to opt out of the study at any time after enrollment.

\section{Consent for publication}

Not applicable.

\section{Competing interests}

The authors declare that they have no competing interests. 


\section{Author details}

'Department of Acupuncture, Guang'anmen Hospital, China Academy of Chinese Medical Sciences, No. 5 Beixiange Street, Xicheng District, Beijing, China. ${ }^{2}$ China Academy of Chinese Medical Sciences, Beijing, China.

\section{Received: 8 August 2019 Accepted: 24 April 2020}

Published online: 23 June 2020

\section{References}

1. Nast A, Rosumeck S, Erdmann R, Alsharif U, Dressler C, Werner RN. Methods report on the development of the European evidence-based (S3) guideline for the treatment of acne - update 2016. J Eur Acad Dermatol Venereol. 2016;30:e1-28

2. Zaenglein AL, Pathy AL, Schlosser BJ, Alikhan A, Baldwin HE, Berson DS, et al. Guidelines of care for the management of acne vulgaris. J Am Acad Dermatol. 2016;74:945-73.e33.

3. Harper JC. An update on the pathogenesis and management of acne vulgaris. J Am Acad Dermatol. 2004;51(1):36-8

4. Cordain L, Lindeberg S, Hurtado M, Hill K, Eaton SB, Brandmiller J. Acne vulgaris: a disease of Western civilization. Arch Dermatol. 2002;138:1584-90.

5. Vilar GN, Santos LA, Sobral Filho JF. Quality of life, self-esteem and psychosocial factors in adolescents with acne vulgaris. An Bras Dermatol. 2015;90(5):622-9.

6. Smithard A, Glazebrook C, Williams HC. Acne prevalence, knowledge about acne and psychological morbidity in mid-adolescence: a community-based study. Br J Dermatol. 2015;145(2):274-9.

7. Mallon E, Newton JN, Klassen A, Stewart-Brown SL, Ryan TJ, Finlay AY. The quality of life in acne: a comparison with general medical conditions using generic questionnaires. Br J Dermatol. 1999;140:672-6.

8. Bez Y, Yesilova Y, Kaya MC, Sir A. High social phobia frequency and related disability in patients with acne vulgaris. Eur J Dermatol. 2011;21:756-60.

9. Fox L, Csongradi C, Aucamp M, du Plessis J, Gerber M. Treatment modalities for acne. Molecules. 2016:21:1063.

10. Cao HJ, Yang GY, Wang YY, Liu JP. Acupoint stimulation for acne: a systematic review of randomized controlled trials. Med Acupunct. 2013;25: 173-94.

11. Son BK, Yun Y, Choi IH. Efficacy of ah shi point acupuncture on acne vulgaris. Acupunct Med. 2010;28(3):126-9.

12. Tan EK, Millington GW, Levell NJ. Acupuncture in dermatology: an historical perspective. Int J Dermatol. 2010;48(6):648-52.

13. Dong BQ, Li LZ, Zhang SJ. Acupuncture at back-shu points with long-round needle for 194 cases of acne [in Chinese]. Zhongguo Zhen Jiu. 2011;31:850.

14. Song SJ. Observation on therapeutic effect of ear point blood-letting combined with cupping on Back-shu points for treatment of acne vulgaris [in Chinese]. Zhongguo Zhen Jiu. 2007;27:626-8.

15. Ma C, Sivamani RK. Acupuncture as a treatment modality in dermatology: a systematic review. J Altern Complement Med. 2015;21:520-9.

16. Cao H, Yang G, Wang Y, Liu JP, Smith CA, Luo H, et al. Complementary therapies for acne vulgaris. Cochrane Database Syst Rev. 2015;1:CD009436.

17. Ding SS, Hong SH, Wang C, Guo Y, Wang ZK, Xu Y. Acupuncture modulates the neuro-endocrine-immune network. QJM. 2014;107:341-5.

18. Scheewe S, Pfab F, Huss-Marp J, Gatti A, Fuqin J, Athanasiadis Gl, et al. Influence of acupuncture on type 1 hypersensitivity itch and the wheal and flare response in adults with atopic eczema - a blinded, randomized, placebo-controlled, cross-over trial [in German]. Deutsche Z Akupunktur. 2011:54:37-9.

19. Dworkin RH, Turk DC, Wyrwich KW, Beaton D, Cleeland CS, Farrar JT, et al. Interpreting the clinical importance of treatment outcomes in chronic pain clinical trials: IMMPACT recommendations. J Pain. 2008;9:105-21.

20. Chren MM, Lasek RJ, Quinn LM, Mostow EN, Zyzanski SJ. Skindex, a qualityof-life measure for patients with skin disease: reliability, validity, and responsiveness. J Invest Dermatol. 1996;107(5):707-13.

21. Chren MM, Lasek RJ, Sahay AP, Sands LP. Measurement properties of Skindex-16: a brief quality-of-life measure for patients with skin diseases. J Cutan Med Surg. 2001:5:105-10.

22. Chan AW, Tetzlaff JM, Gøtzsche PC, Altman DG, Mann H, Berlin JA, et al. SPIRIT 2013 explanation and elaboration: guidance for protocols of clinical trials. BMJ. 2013;346:e7586.

23. MacPherson $\mathrm{H}$, Altman DG, Hammerschlag R, Youping L, Taixiang W, White A, et al. Revised STandards for Reporting Interventions in Clinical Trials of
Acupuncture (STRICTA): extending the CONSORT statement. PLoS Med. 2010;: :e1000261.

24. Descamps V. Clinical guidelines for management of acne vulgaris [letter]. JAMA. 2017:317(2):213.

25. Doshi A, Zaheer A, Stiller MJ. A comparison of current acne grading systems and proposal of a novel system. Int J Dermatol. 2010;36(6):416-8.

26. Ramli R, Malik AS, Hani AF, Jamil A. Acne analysis, grading and computational assessment methods: an overview. Skin Res Technol. 2012:18:1-14.

27. Huang LX, Zhao JS, Wu ZC, Wu XD, Tan YS. Editorial explanation on the state standard The Name and Location of Acupoints (2006, edition) [in Chinese]. Zhongguo Zhen Jiu. 2009;29(11):924-6.

28. Xiang L. Guideline for diagnosis and treatment of acne (the 2014 revised edition) [in Chinese]. J Clin Dermatol. 2015;1:52-7.

29. Finlay AY, Khan GK. Dermatology Life Quality Index (DLOI)—a simple practical measure for routine clinical use. Clin Exp Dermatol. 1994;19:210-6.

30. Basra MK, Salek MS, Camilleri L, Sturkey R, Finlay AY. Determining the minimal clinically important difference and responsiveness of the Dermatology Life Quality Index (DLQI): further data. Dermatology. 2015; 230:27-33.

31. Palli MB, Reyes-Habito CM, Lima XT, Kimball AB. A single-center, randomized double-blind, parallel-group study to examine the safety and efficacy of $3 \mathrm{mg}$ drospirenone/0.02 mg ethinyl estradiol compared with placebo in the treatment of moderate truncal acne vulgaris. J Drugs Dermatol. 2013;12:633-7.

32. Reich A, Heisig M, Phan NQ, Taneda K, Takamori K, Takeuchi S, et al. Visual analogue scale: evaluation of the instrument for the assessment of pruritus. Acta Derm Venereol. 2012;92:497-501.

33. Kim K, Kim K, Lee J. Inhibitory effects of Cheongsangbangpoong-tang on both inflammatory acne lesions and facial heat in patients with acne vulgaris: a randomized controlled trial protocol. BMC Complement Altern Med. 2016;16:21.

34. Uhlenhake E, Yentzer BA, Feldman SR. Acne vulgaris and depression: a retrospective examination. J Cosmet Dermatol. 2010;9:59-63.

35. Bhate K, Williams HC. Epidemiology of acne vulgaris. Br J Dermatol. 2012; 168:474-85.

36. Gupta M. The development of an acne quality of life scale: reliability, validity, and relation to subjective acne severity in mild to moderate acne vulgaris. Acta Derm Venereol. 1998;78:451-6.

37. Shi GX, Yang XM, Liu CZ, Wang LP. Factors contributing to therapeutic effects evaluated in acupuncture clinical trials. Trials. 2012:13:42.

38. Vickers AJ. Placebo controls in randomized trials of acupuncture. Eval Health Prof. 2002;25(4):421-35.

39. Liu B, Xu H, Ma R, Mo Q, Yan S, Liu Z. Effect of blinding with a new pragmatic placebo needle: a randomized controlled crossover study. Medicine (Baltimore). 2014:93:e200

40. Vincent CA. A controlled trial of the treatment of migraine by acupuncute. Clin J Pain. 1989:5:305-12.

41. Olausson H, Wessberg J, Morrison I, McGlone F, Vallbo A. The neurophysiology of unmyelinated tactile afferents. Neurosci Biobehav Rev. 2010;34:185-91.

\section{Publisher's Note}

Springer Nature remains neutral with regard to jurisdictional claims in published maps and institutional affiliations.

\section{Ready to submit your research? Choose BMC and benefit from:}

- fast, convenient online submission

- thorough peer review by experienced researchers in your field

- rapid publication on acceptance

- support for research data, including large and complex data types

- gold Open Access which fosters wider collaboration and increased citations

- maximum visibility for your research: over $100 \mathrm{M}$ website views per year

At BMC, research is always in progress.

Learn more biomedcentral.com/submission 